\title{
BUSCANDO A FORMA PELO CONTEXTO GEOGRÁFICO 1
}

\author{
SEEKING THE SHAPE BY GEOGRAPHICAL CONTEXT \\ Jarryer Andrade De Martino \\ Centro Universitário Barão de Mauá (CBM) \\ jarryermartino@gmail.com
}

\begin{abstract}
Resumo
O processo de projeto aceita diferentes estratégias para a obtenção da sua solução, o que difere uma das outras são os procedimentos adotados por cada projetista, assim como os tópicos escolhidos para serem explorados em cada projeto. Quando a estratégia projetual incorpora ferramentas digitais no processo é possível antever os resultados através da simulação, gerando informações sobre a eficiência das soluções capazes de direcionar o projeto para adequações ou novas soluções. Esse processo pode ser automatizado ao adotar o sistema generativo evolutivo como método de projeto, uma vez que ele apresenta um mecanismo de busca por soluções que atenda simultaneamente e satisfatoriamente os diferentes critérios estabelecidos pelo projetista, definindo a técnica form-finding. O artigo tem como objetivo apresentar três estudos de projetos modelados a partir das informações dos seus locais de implantação, sendo adotado como método de projeto o sistema generativo evolutivo como uma técnica form-finding. A apresentação consiste em estruturar algoritmicamente o método evolutivo, focando a utilização dos dados climáticos como critérios norteadores do desenvolvimento do processo de projeto.
\end{abstract}

Palavras-chave: Algoritmo evolutivo. Sistema generativo. Método de projeto. Insolação.

\begin{abstract}
The design process supports different strategies for obtaining its solution; the difference among them is the procedures adopted by each designer; the topics chosen to be explored in each project. When design strategy incorporates digital tools in the process it is possible to predict the results by simulation, generating information about efficiency of the solutions which are capable to lead the project for some adjustments or new solutions. The evolutionary method as generative design system can automate that process, because it has a search engine to find the best solution that satisfying simultaneously different criteria established by the designer, setting the formfinding technique. The article aims to present three design studies modeled from the information of their deployment sites; the evolutionary generative system was adopted as a form-finding technique. The structure algorithmic of evolutionary method is presented, focusing on the use of climate data as criteria guiding the design process.
\end{abstract}

Keywords: Evolutionary algorithm. Generative system. Design method. Insolation.

\section{INTRODUÇÃO}

O processo de projeto aceita diferentes estratégias para a obtenção da sua solução, o que difere uma das outras são os procedimentos adotados por cada projetista, assim como os tópicos escolhidos para serem explorados em cada projeto. Quando esses tópicos estão

\footnotetext{
${ }_{1}^{1}$ MARTINO, J. A. Buscando a forma pelo contexto geográfico. In: ENCONTRO BRASILEIRO DE TECNOLOGIA DE INFORMAÇÃO E COMUNICAÇÃO NA CONSTRUÇÃO, 7., 2015, Recife. Anais... Porto Alegre: ANTAC, 2015.
} 
relacionados ao sítio onde o projeto será implantando, as soluções obtidas são mais coerentes e precisas com o seu contexto geográfico. Isso amplia as possibilidades exploratórias do projeto, permitindo adotar abordagens como o da eficiência energética e a melhor integração entre o exterior e interior, favorecendo os aspectos físicos e psicológicos dos usuários no ambiente construído. A história nos mostra que, com experiência e imaginação, a arquitetura do abrigo evoluiu criando soluções diversificadas para enfrentar os desafios climáticos, reconhecendo a adaptação climática regional como um princípio para a arquitetura.

As ferramentas digitais assumiram um papel fundamental nesse contexto, pois elas permitem antever resultados através da simulação, gerando informações sobre a eficiência da solução adotada que serão capazes de direcionar o processo de projeto. Assim, as informações obtidas a partir do próprio modelo geométrico possibilitam as adequações necessárias para redefinir o projeto ou gerar novas soluções. Atualmente vivemos uma era onde a tecnologia é manipulada e moldada às nossas necessidades, sendo criadas novas situações que até então não existiam ou não tinham demanda, permitindo a adequação ou criação de ferramentas e processos que redefinem o método de projeto. Assim, o distanciamento entre o usuário e os meios digitais está cada vez mais diluído, sendo atribuídas aptidões específicas a cada um, fortalecendo a relação de parceria durante o processo de desenvolvimento projetual (TERZIDIS, 2009). Isso dá origem a um espaço único, integrado e potencializado, possibilitando ao projetista navegar fluidamente entre os diferentes territórios disciplinares. Exemplo disso é a incorporação no método de projeto do pensamento algorítmico, da computação, da análise do desempenho como critério orientador no desenvolvimento projetual e da utilização de conceitos da engenharia como a otimização (não apenas como estratégia orçamental, mas também nas estratégias formais e organizacionais), permitindo descobrir e criar novas técnicas (KOLAREVIC, 2009). Além disso, as técnicas digitais também passaram a influenciar a documentação, fabricação e montagem dos edifícios, propiciando a emergência de uma arquitetura que reflete a era digital, apresentando alto nível de complexidade, capacitando uma maior sensibilidade, e respondendo as exigências dos aspectos contextuais como o lugar, o programa e a intenção expressiva (OXMAN, 2006).

Segundo Lorenzo-Eiroa e Sprecher (2013), a capacidade oferecida pelas tecnologias da informação possibilita redefinir os modos de produção e a natureza de expressão da arquitetura, permitindo fazer a transição de estruturas rígidas em sistemas abertos. Assim, a condição de causalidade é transformada em uma situação não linear, recebendo a influência simultânea de uma grande variedade de informações que representam uma realidade em constante mutação: uma realidade moldada pela potencialidade, instabilidade e probabilidade. O método de projeto que reflete essa condição é o sistema generativo, pois ele consiste de um conjunto de instruções operativas, ou seja, uma definição algorítmica responsável pela geração de soluções potenciais, apresentando uma estruturação lógica capaz de solucionar um determinado problema em diferentes situações, gerando um complexo espaço de soluções (TERZIDIS, 2009). Isso resulta em um método indireto de projeto, uma vez que o projetista não interage diretamente com o objeto projetado, mas sim na definição e estruturação do sistema generativo (FISCHER; HERR, 2004). Segundo Kolarevic (2009), no projeto de arquitetura contemporânea avant-garde, vários processos generativos e de produção digital estão se abrindo para novos territórios para conceituar a articulação morfológica arquitetônica, focado na emergência e propriedades adaptativas da forma. Isso pode ser verificado na ênfase de alguns modelos digitais de projeto, alterando o processo de "fazer a forma" para o de "encontrar a forma", sendo intencionalmente utilizadas técnicas generativas para ajudar o projetista a obter uma forma (form- finding).

Dessa forma, este artigo tem como objetivo apresentar o sistema generativo evolutivo como um método de projeto capaz de ser manipulado como uma técnica form-finding, utilizando o 
mecanismo evolutivo para encontrar as soluções melhores ajustadas aos critérios estabelecidos pelo projetista e que adotam como referência o contexto geográfico de implantação do projeto. Para isso, são apresentados três estudos de projetos modelados a partir das informações dos seus locais de implantação.

\section{SISTEMA GENERATIVO EVOLUTIVO E O CONTEXTO GEOGRÁFICO}

O principal enfoque para a sua utilização envolve a busca por soluções para problemas que atendam em maior ou menor grau satisfatoriamente e simultaneamente todos os objetivos que the são exigidos, configurando um problema com multiobjetivos (FLOREANO; MATTIUSSI, 2008). Neste caso, o problema é composto por diferentes variáveis que precisam ter os seus valores negociados, obtendo soluções que consigam manter o equilíbrio e não priorizem apenas uma delas (BENTLEY, 1999; MITCHELL, 1999). Dessa forma, as diferentes possibilidades de negociação entre as variáveis definem uma variedade de soluções para o mesmo problema, compondo o que pode ser chamado de "espaço de soluções". Os principais componentes dos algoritmos são a representação dos indivíduos, as populações, a função de avaliação (fitness function) que dará origem ao valor de fitness (valor que cada solução recebe como se fosse uma nota), o mecanismo de seleção, os operadores de diversidade (mutação e recombinação) e o tempo de duração ou o número de gerações dos algoritmos (número de ciclos). A partir desses componentes é possível incorporar a ideia de reprodução com herança genética, a variação aleatória em uma população de indivíduos e a simulação de uma seleção natural para a obtenção da próxima geração (FLOREANO; MATTIUSSI, 2008), permitindo assim a simulação do processo evolutivo teorizado por Darwin.

Dessa forma, a integração do método evolutivo com a simulação computacional é capaz de auxiliar o projetista na obtenção de formas mais eficientes desde a fase inicial de concepção projetual, configurando um método com características da técnica form-finding. A simulação computacional do desempenho das soluções permite a obtenção de dados que irão alimentar o mecanismo evolutivo, permitindo avaliar, classificar e selecionar as soluções que melhor satisfazem os critérios definidos pelo projetista. Assim, o processo de definição formal é automatizado, sendo geradas volumetrias a partir da avaliação do seu desempenho diante do contexto geográfico a ser implantado. Isso permitirá uma arquitetura ecologicamente sensível, e que segundo Yeang (1994), faz com que o processo de projeto arquitetônico deixa de ser uma mera arte extravagante, e assuma o potencial de uma ciência confiável, adotando uma metodologia embasada na pesquisa, desenvolvimento e design. Para ele, existem quatro justificativas fundamentais para esse tipo de projeto, (1) a redução do consumo de energia durante a fase de operação do edifício, (2) a possibilidade de oferecer bem estar ao usuário, permitindo usufruir do clima externo local, (3) a questão ecológica, explorando os dados meteorológicos do local a fim de se obter a eficiência e menos dependência dos combustíveis fósseis, e (4) a possibilidade de estabelecer o regionalismo arquitetônico, caracterizada pela adaptação climática regional, assumindo maior ajuste ao seu contexto geográfico.

\section{ESTUDOS DE PROJETOS}

Os estudos apresentados correspondem à exercícios de projetos desenvolvidos para verificar a implementação do algoritmo evolutivo como método de projeto, adotando os dados informacionais obtidos a partir da simulação do desempenho das soluções em seu contexto geográfico como referência para a definição formal. Para isso, o sistema generativo evolutivo foi estruturado em três blocos, o primeiro é responsável pela definição dos elementos que compõem a forma e as suas relações paramétricas, o segundo está relacionado com os algoritmos evolutivos e os critérios para a restrição do campo de 
soluções, e o terceiro corresponde à geração de dados a partir da simulação climática conforme o contexto geográfico (Figura 1).

Figura 1 - Estruturação do sistema generativo evolutivo

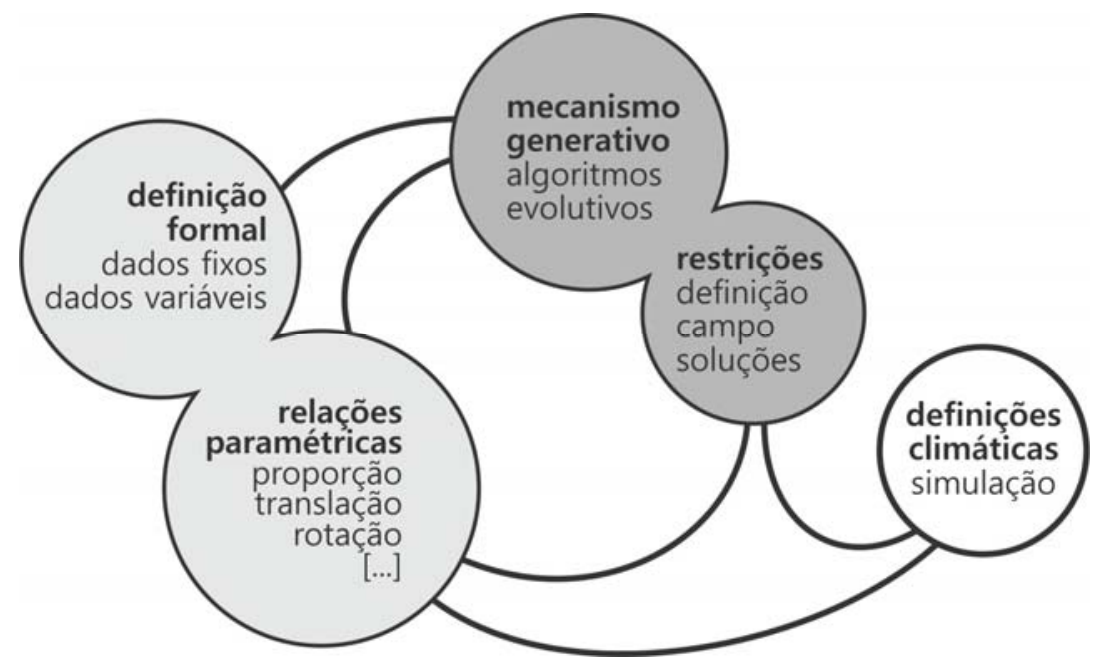

Fonte: elaborado pelo autor.

O sistema foi implementado computacionalmente utilizando o editor gráfico de algoritmo Grasshopper $®$, plugin para o programa Rhinoceros $®$ da McNeel. Isso possibilitou a criação de um sistema automatizado para a geração e busca por soluções que melhor atendam simultaneamente e satisfatoriamente os diferentes critérios definidos como referência. Os estudos de projeto apresentados neste artigo possuem como critérios as definições formais e o desempenho da solução mediante a sua simulação no contexto geográfico a ser implantado.

\subsection{Estudo de projeto 1: criação de uma cobertura}

Este estudo de projeto corresponde à criação de uma cobertura complementar a uma edificação existente, mantendo o sombreamento de uma área já coberta, mas que recebe insolação no período das $14 \mathrm{~h}$ às $16 \mathrm{~h}$ devido a uma abertura lateral. Por isso, o estudo considerou o solstício de verão na data de 23 de dezembro de 2013, às 16h, horário em que ocorre a maior incidência solar na região especificada. $O$ desenho da estrutura apresenta aberturas ao longo da sua superfície, contribuindo para a ventilação natural. Assim, a solução ideal para este projeto deverá satisfazer aos critérios de (1) maximização da área de sombreamento e do (2) somatório das áreas de aberturas, duas variáveis contraditórias a serem negociadas.

A facilidade de manipulação do sistema generativo evolutivo, devido à automatização do processo, possibilitou realizar cinco experimentos, sendo adotadas duas abordagens para a concepção da cobertura, uma parcialmente parametrizada (com variação apenas no dimensionamento das aberturas) e a outra totalmente parametrizada (permitindo variações na forma da superfície e o dimensionamento das aberturas) (Figura 2). A primeira abordagem foi empregada nos quatro primeiros experimentos, sendo utilizadas duas formas como referência para a superfície da cobertura, uma curvilínea e outra linear. Cada uma delas foi testada considerando duas diferentes orientações para as aberturas (Norte e Oeste). Os resultados obtidos nos experimentos parcialmente parametrizados permitiram verificar que as aberturas orientadas para o Norte apresentaram melhores índices, sendo esta orientação adotada para a realização da segunda abordagem, ou seja, a totalmente parametrizada. O experimento EX5 apresentou uma superfície de referência definida por 
curvas parametrizadas controladas automaticamente pelo algoritmo evolutivo, permitindo buscar e testar a eficiência das diferentes soluções.

Figura 2 - Abordagens dos experimentos
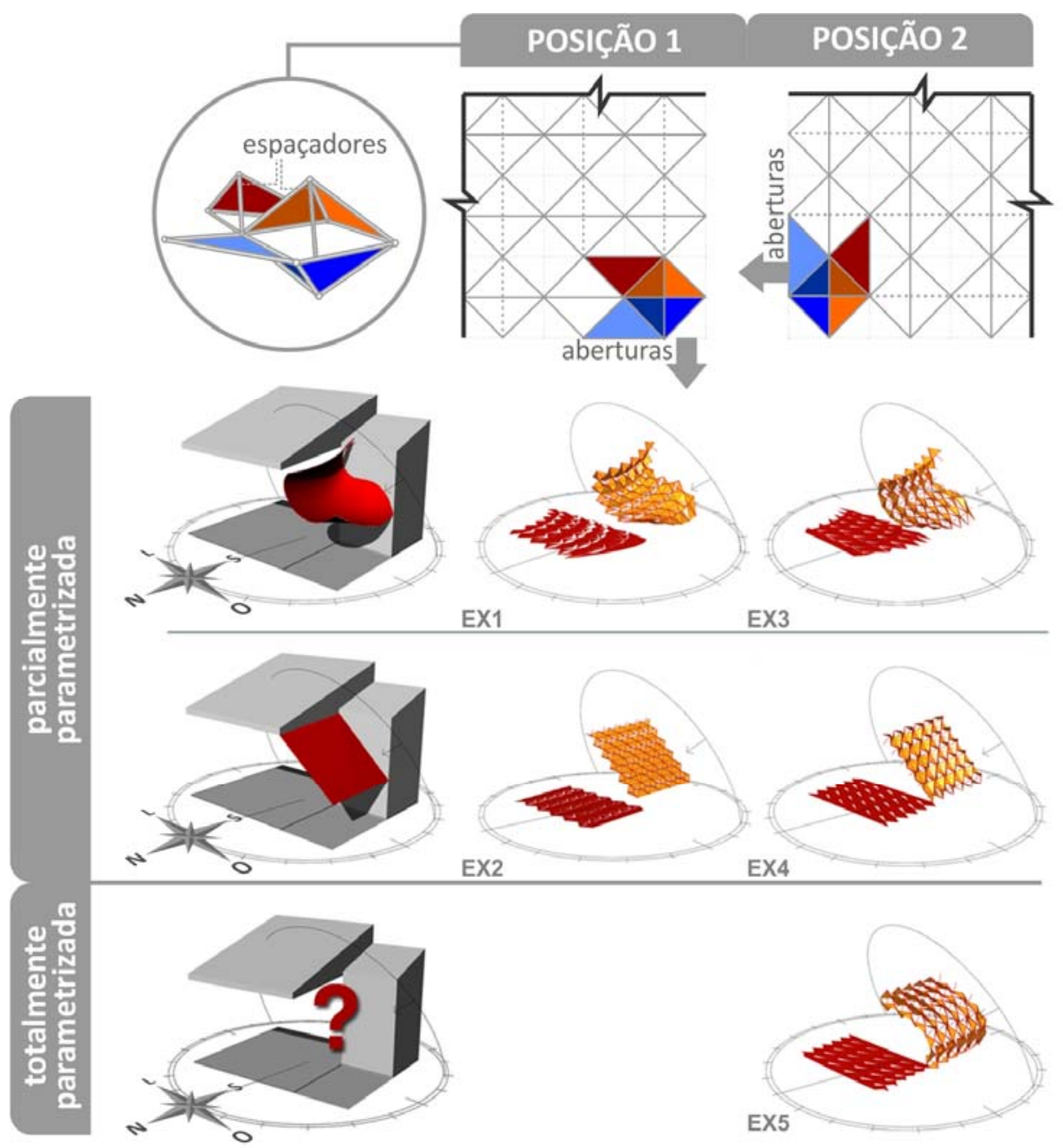

Fonte: elaborado pelo autor.

As três superfícies utilizadas como referência para a definição formal da cobertura foram transformadas em uma malha constituída por setenta e dois módulos, sendo ela a base para a construção de uma estrutura tridimensional triangulada. Os vértices e as mediatrizes das laterais dos módulos foram adotados como pontos de conexão dos elementos estruturais. Alguns desses elementos são os responsáveis pelo dimensionamento das aberturas (os espaçadores), e foram posicionados em todos os módulos da estrutura triangular (Figura 2). O tamanho dos espaçadores varia em função da distância entre a sua posição na malha e o ponto de atração mais próximo. Desta forma, dois pontos de atração, movidos independentemente e automaticamente pelo algoritmo evolutivo foram distribuídos na superfície, explorando diferentes posições e gerar diversidade nas dimensões das aberturas (Figura 3). Isso garantiu maior flexibilidade ao sistema, ajudando o algoritmo evolutivo a encontrar as melhores soluções para o problema, pois quanto maior for a distância entre os espaçadores e os pontos, maiores serão os espaçamentos gerados nos vãos, e vice-versa. 
Figura 3 - Pontos de atração regulando a dimensão dos espaçadores

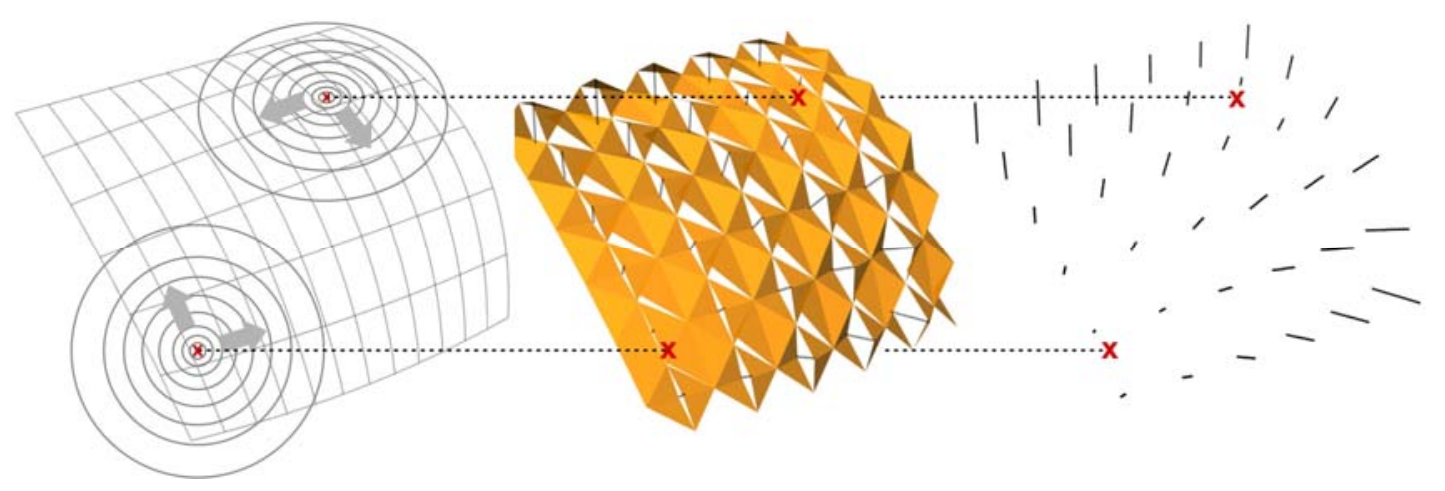

Fonte: elaborado pelo autor.

Os valores de aptidão obtidos permitiram identificar quais dos experimentos e a melhor solução que atendem aos objetivos desejados, o EX5 é o que apresenta o maior valor, aumentando o seu valor de fitness em $9,6 \%$, enquanto que os outros variaram entre $0,3 \%$ e $2,5 \%$ (Figura 4). Com relação à área de sombreamento, o EX5 apresentou um acréscimo de $10,2 \%$ na área final, já os outros experimentos ficaram entre $0,46 \%$ e 1,66\% (o EX1 possuiu um decréscimo de $8,29 \%$ ). No somatório dos comprimentos dos vãos, o experimento EX5 apresentou um acréscimo de apenas $5,2 \%$, taxa inferior quando comparada aos valores de $42,3 \%$ do EX1 e $11,5 \%$ do EX3, mas que provavelmente justifica possuírem a menor área de sombreamento. Como no sistema generativo evolutivo as melhores soluções são aquelas que apresentam o maior valor de fitness, ao analisar a Figura 4 é possível verificar que o EX5 se destaca com relação aos outros. Além disso, o EX5 é o que apresenta o melhor equilíbrio entre os diferentes objetivos a serem atingidos, mesmo apresentando uma taxa inferior ao obtido no EX1 e EX3 para o somatório do comprimento dos vãos, isso corresponde a uma diferença muito pequena ao comparar com o ganho obtido na área de sombreamento.

Figura 4 - Valores de fitness dos diferentes experimetos

\section{Fitness}

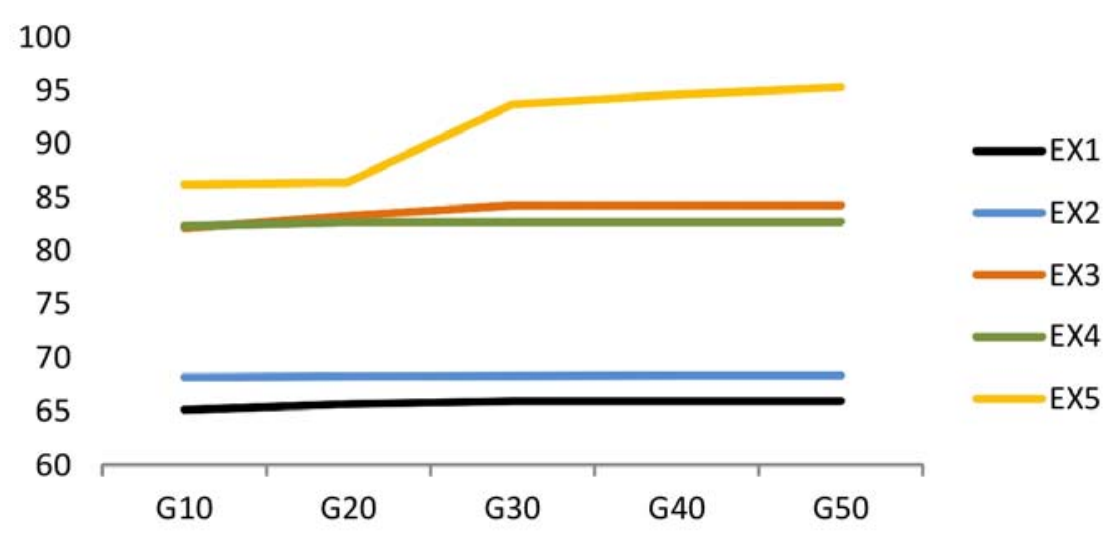

Fonte: elaborado pelo autor.

Os indivíduos dos experimentos EX1 e EX3 possuem o mesmo valor de 12,4m para o somatório dos comprimentos dos vãos, enquanto o EX5 atingiu $11,9 \mathrm{~m}$, valor não muito 
distante dos outros experimentos. Com relação a área de sombreamento o distanciamento entre os valores obtidos nos experimentos é maior, o EX5 atingiu o melhor resultado com a área de $27,8 \mathrm{~m}^{2}$, enquanto que o EX1 e EX3 apresentaram os valores de $17,8 \mathrm{~m}^{2}$ e $24,1 \mathrm{~m}^{2}$ respectivamente, fazendo deste critério o determinante para a escolha da melhor solução (Figura 5).

Figura 5 - Comparativo entre a forma inicial e a final encontrada pelo Sistema Generativo Evolutivo do EX5

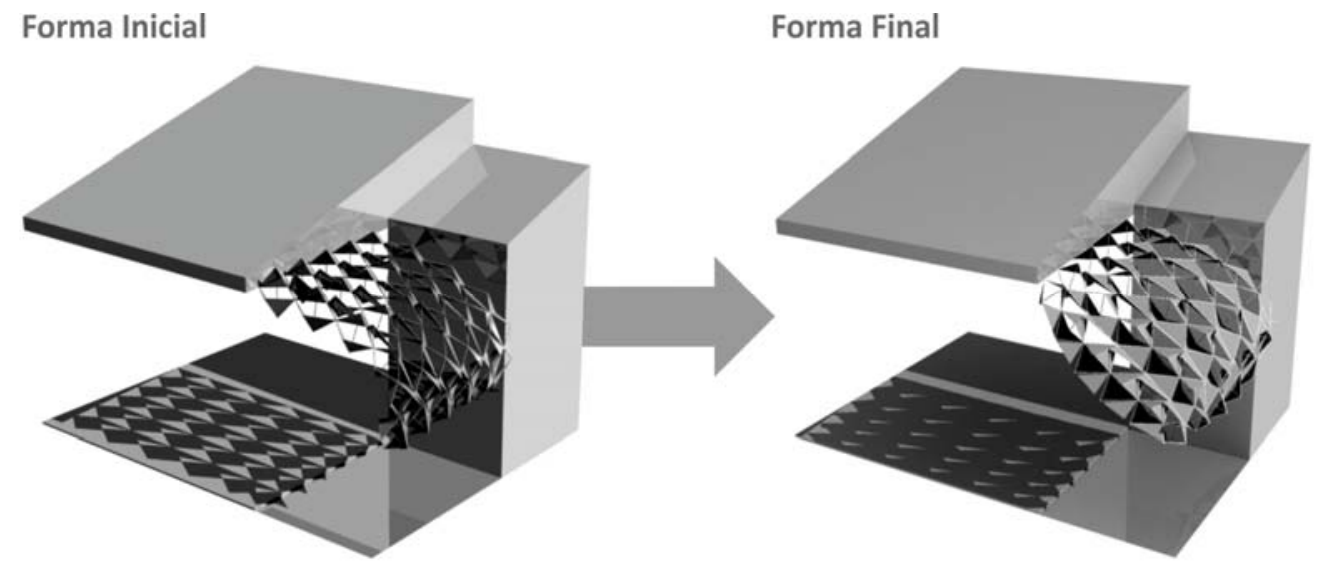

Fonte: elaborado pelo autor.

É importante perceber que a solução emergiu (Figura 5) a partir da intensão de negociar e maximizar os valores de ambos os critérios (área de sombra e somatório dos comprimentos dos vãos), sendo todo o processo embasado nas informações obtidas em simulações digitais sobre o desempenho das soluções no local a ser implantado.

\subsection{Estudo de projeto 2: modelagem volumétrica de edifício}

Neste estudo de projeto o objetivo foi modelar volumetricamente um edifício conforme as condições climáticas do local a ser implantado, adotando como critérios a (1) a distribuição de forma mais homogênea possível da insolação e a (2) sua maximização nas superfícies dos edifícios. Dessa forma, seria possível definir desde a fase de concepção, uma volumetria favorável à implementação nas fachadas de mecanismos responsáveis pelo aproveitamento energético, criando condições para a captação energética. Foram desenvolvidos três experimentos, cada um apresentando diferentes configurações para o sistema generativo evolutivo, duas abordagens parcialmente parametrizadas (com variação apenas na sua volumetria) e a um totalmente parametrizada (permitindo variações na implantação e volumetria do edifício). Os dois primeiros experimentos (EX1 e EX2) utilizaram a abordagem parcialmente parametrizada, sendo testadas variações nos critérios de avaliação e seleção das soluções. Os critérios que melhor apresentaram resultados nestes experimentos foram incorporados no terceiro (EX3), sendo estruturado conforme a segunda abordagem.

Para a realização do estudo foi definido um modelo paramétrico tridimensional do edifício, composto por três polígonos sobrepostos, cada um contendo três lados e circunscritos em uma circunferência de $16 \mathrm{~m}$ de raio (Figura 6). O primeiro foi posicionado na base (definindo a implantação do edifício), o segundo na porção central ( $24 \mathrm{~m}$ de altura do primeiro), e o terceiro no topo do volume (48m de altura com relação à base). O EX1 e o EX2 não apresentaram variações no polígono da base, definindo assim, uma implantação fixa durante todo o processo de execução do algoritmo evolutivo, a variação na rotação ocorreu apenas no EX3. O polígono intermediário e o do topo variaram nos três experimentos, 
sofrendo transformações no tamanho (através de um fator de escala entre 0,5 e 1,5) e na sua rotação (variando entre $0^{\circ}$ e $120^{\circ}$, com valores pares para o polígono intermediário e ímpares para o do topo), sendo ambas variáveis controladas automaticamente pelos algoritmos evolutivos, permitindo a torsão da volumetria para a provável adaptação ao recebimento da luz solar.

Figura 6 - Modelo paramétrico do edifício

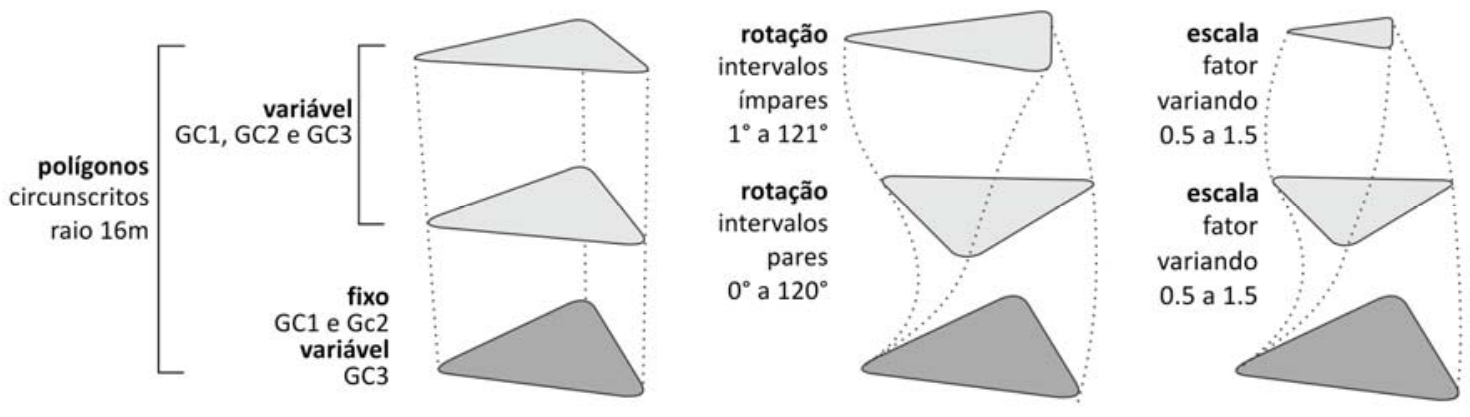

Fonte: elaborado pelo autor.

Todos os experimentos apresentaram como configuração inicial do volume uma rotação de $120^{\circ}$ e o fator de escala igual a 0,5 para o polígono intermediário, para o do topo foi definida a rotação de $121^{\circ}$ e o fator de escala de 0,6 , gerando uma torção na volumetria. A configuração padrão adotada para os algoritmos evolutivos dos três experimentos considerou a maximização do valor de aptidão, ou seja, o valor máximo para a taxa de insolação sobre as superfícies do edifício (medida em $\mathrm{Wh} / \mathrm{m}^{2}$ ).

Para a análise dos resultados foi adotada a solução com o maior valor de aptidão encontrada na última geração do algoritmo evolutivo de cada experimento, sendo utilizada a avaliação visual para a verificação da distribuição da carga energética nas superfícies do edifício. Esta avaliação pode ser realizada visualmente utilizando a escala cromática gerada pelo Ecotect, que associa a cada cor um valor de carga energética.

Figura 7 - Modelo paramétrico do edifício

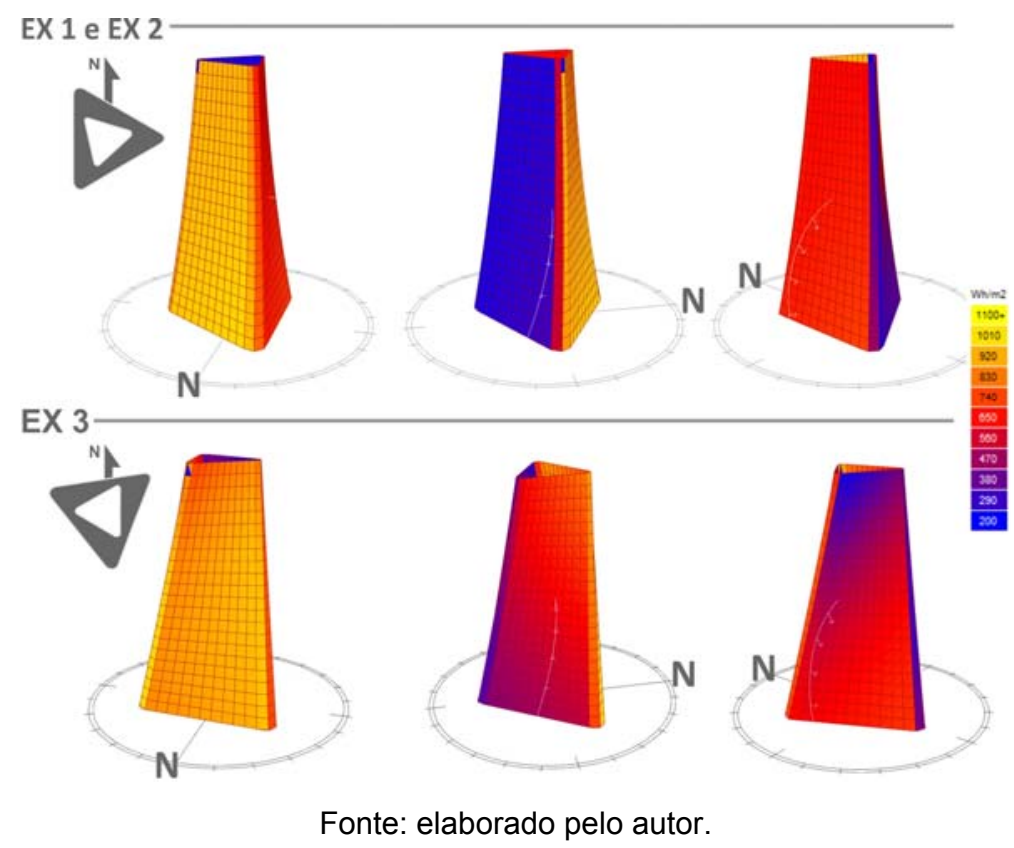


No EX1 e EX2 é possível verificar visualmente que mesmo diante da possibilidade de torção do edifício, não foi possível obter um resultado formal capaz de uma distribuição mais homogênea da insolação sobre a superfície (Figura 7). Isso ocorreu porque as torções geravam em uma mesma superfície regiões com o máximo de sombreamento e o máximo de insolação, criando valores muito distantes entre o mínimo e o máximo. Dessa forma, o algoritmo evolutivo não conseguiu encontrar soluções que apresentassem equilíbrio entre essas variáveis, impossibilitando-o de encontrar as soluções que satisfizessem os critérios e permitisse a sua evolução. Dessa forma, percebeu-se que os parâmetros de ecala e rotação nos polignos da região central e do topo do edifício não eram suficientes para gerar soluções que satisfizessem os critérios desejados. Por isso, foi realizado um terceiro experimento (EX3) considerando a rotação da base do edifício, permitindo a redefinição da sua implantação, aumentando o campo de soluções para a obtenção de outras possibilidades formais, gerando maior flexibilidade. Embora o resultado obtido ainda apresenta áreas de sombreamento, elas correspondem à regiões menores e distribuídas em diferentes superfícies da volumetria do edifício. É interessante perceber que o resultado formal da volumetria é muito semelhante aos obtidos no EX1 e no EX2, ficando a diferença apenas pela sutil rotação no topo e o reposicionamento da implantação do edifício, sofrendo uma rotação com relação ao Norte (Figura 7). Essas pequenas diferenças foram suficientes para ajustar o edifício ao seu contexto geográfico, passando a oferecer um resultado mais eficiente quanto à distribuição mais homogênea da insolação nas superfícies do edifício e a sua maximização conforme ao atendimento simultâneo desses critérios.

Figura 8 - Comparativo entre a forma inicial e a final encontrada pelo Sistema Generativo Evolutivo do EX3

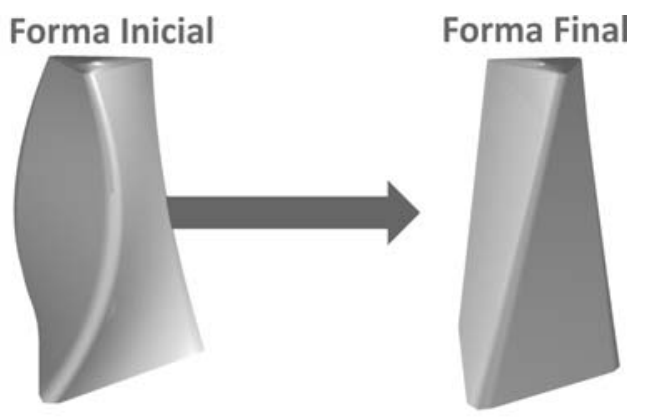

Fonte: elaborado pelo autor.

Na Figura 8 é possível verificar a transformação da forma inicial ao comparar com o resultado final obtido pelo sistema generativo evolutivo. Essa transformação buscou de maneira automatizada a constante adequação das soluções ao atendimentos dos critérios definidos como os ideais para este projeto, ou seja, a distribuição mais homogênea e a maximização da insolação sobre as superfícies do edifício.

\subsection{Estudo de projeto 3: modelagem volumétrica de edifício}

Este último estudo de projeto é semelhante ao caso anterior, os critérios a serem avaliados pelo algoritmo evolutivo são: (1) a maior área de ocupação no lote, respeitando os recuos e o gabarito previamente definido (escalonamento), (2) o maior valor para o volume, (3) a menor dimensão para o perímetro e (4) a maximização da incidência solar. Para isso, foi definido um modelo paramétrico composto por seis módulos independentes que se deslocam livremente pelo lote com dimensão de $20 \mathrm{~m}$ de largura por $50 \mathrm{~m}$ de comprimento (Figura 9). Cada módulo possui a forma de um quadrado com dimensão de $10 \mathrm{~m}$, com altura variando entre $8 \mathrm{~m}$ e $40 \mathrm{~m}$, conforme a sua posição no lote, sendo possível a sobreposição deles. Assim, o lote foi zoneado de maneira a apresentar alturas específicas para os 
módulos de acordo com cada zona, definindo uma volumetria escalonada (Figura 9).

Figura 9 - Zoneamento do lote para definição das alturas dos módulos
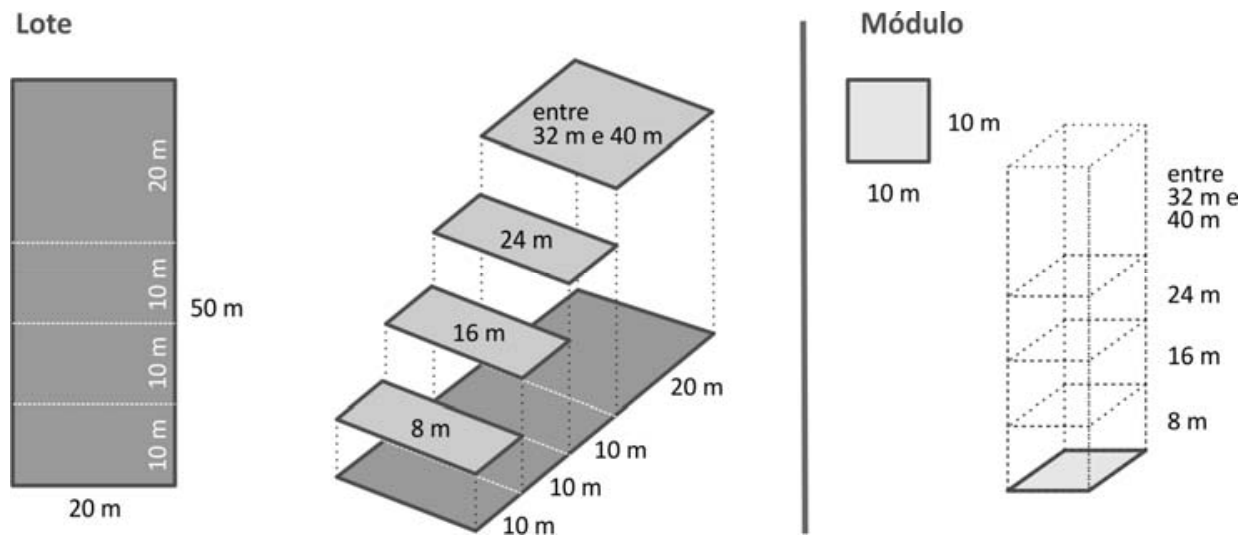

Fonte: elaborado pelo autor.

A partir deste modelo paramétrico foram realizados três experimentos que se diferenciam pela redefinição da função de avaliação (fitness function), criando diferentes estratégias para a busca da maximização dos índices de insolação (Figura 10). No EX1 a maximização adotou como referência a media aritmética dos valores da lista dos índices, o EX2 utilizou como estratégia a seleção de um valor na posição central dessa lista, forçando os algoritmos evolutivos a encontrarem soluções com maiores valores nessa região que não é estável (devido à variação formal do projeto), e no EX3 foi escolhida aleatoriamente a sexta posição da lista de índices, evitando o primeiro valor que seria igual em ambos os experimentos, uma vez que sempre existirá algum ponto com zero de insolação. A adoção dessas três formas de avaliação surgiu do interesse de verificar quais delas é o processo mais eficiente para a obtenção da maior insolação na superfície do edifício.

Figura 10 - Volumetria e dados obtidos nos experimentos

\begin{tabular}{|c|c|c|c|c|c|c|c|c|c|}
\hline \multicolumn{4}{|c|}{$\begin{array}{l}\text { [a] Média } \\
\text { dos Índices }\end{array}$} & \multicolumn{3}{|c|}{$\begin{array}{l}{[b] \text { Valores na }} \\
\text { Posição Central }\end{array}$} & \multicolumn{3}{|c|}{$\begin{array}{l}\text { [c] Valores na } \\
\text { Sexta Posição }\end{array}$} \\
\hline & EX7 & EX8 & EX9 & EX7 & EX8 & EX9 & EX7 & EX8 & EX9 \\
\hline somatória & 68121,96 & 65804,11 & 77230,03 & 176,37 & 160,72 & 151,71 & 0 & 0 & 0 \\
\hline células & 287 & 299 & 321 & 176,64 & 168,23 & 151,71 & 60,8 & 60,8 & 60,8 \\
\hline \multirow[t]{16}{*}{ média } & 237 & 220 & 241 & 177,05 & 168,23 & 152,23 & 60,8 & 60,8 & 60,8 \\
\hline & & & & 179,33 & 168,23 & 152,95 & 61,28 & 60,8 & 63,21 \\
\hline & & & & 180,46 & 170,46 & 153,9 & 63,21 & 60,8 & 63,21 \\
\hline & & & & 181,74 & 171,44 & 154,66 & 63,21 & 60,88 & 63,24 \\
\hline & & & & 183,16 & 175,7 & 163,78 & 63,21 & 61,2 & 63,29 \\
\hline & & & & 183,82 & 179,13 & 167,73 & 63,24 & 61,42 & 65,05 \\
\hline & & & & 184,5 & 181,65 & 169,48 & 67,11 & 61,68 & 67,1 \\
\hline & & & & 184,92 & 186,63 & 169,48 & 69,75 & 61,68 & 67,72 \\
\hline & & & & 186,29 & 187,82 & 171,06 & 74,76 & 61,71 & 68,46 \\
\hline & & & & 188,02 & 191,95 & 173,4 & & & \\
\hline & & & & 189,85 & 192,15 & 173,75 & & & \\
\hline & & & & 192,77 & 192,77 & 174,02 & & & \\
\hline & & & & 192,91 & $192,77[$ & 174,06 & & & \\
\hline & & & & 193,27 & 193,41 & 176,03 & & & \\
\hline & & & & 193,43 & 193,41 & 177,07 & & & \\
\hline & & & & 195,18 & 194,12 & 180,16 & & & \\
\hline
\end{tabular}

Fonte: elaborado pelo autor.

O experimento EX3 gerou o resultado mais eficiente, apresentando uma volumetria com até $13 \%$ a mais de superfície com o maior carga energética $\left(601-700 \mathrm{Wh} / \mathrm{m}^{2}\right)$, ou seja, a solução volumétrica com a maior insolação na sua superfície. A estratégia adotada para este 
experimento forçou o mecanismo evolutivo a buscar e selecionar as soluções que melhor satisfizessem os seus critérios, obtendo soluções formais diferenciadas (Figura 11).

Figura 11 - Volumetria e dados obtidos nos experimentos

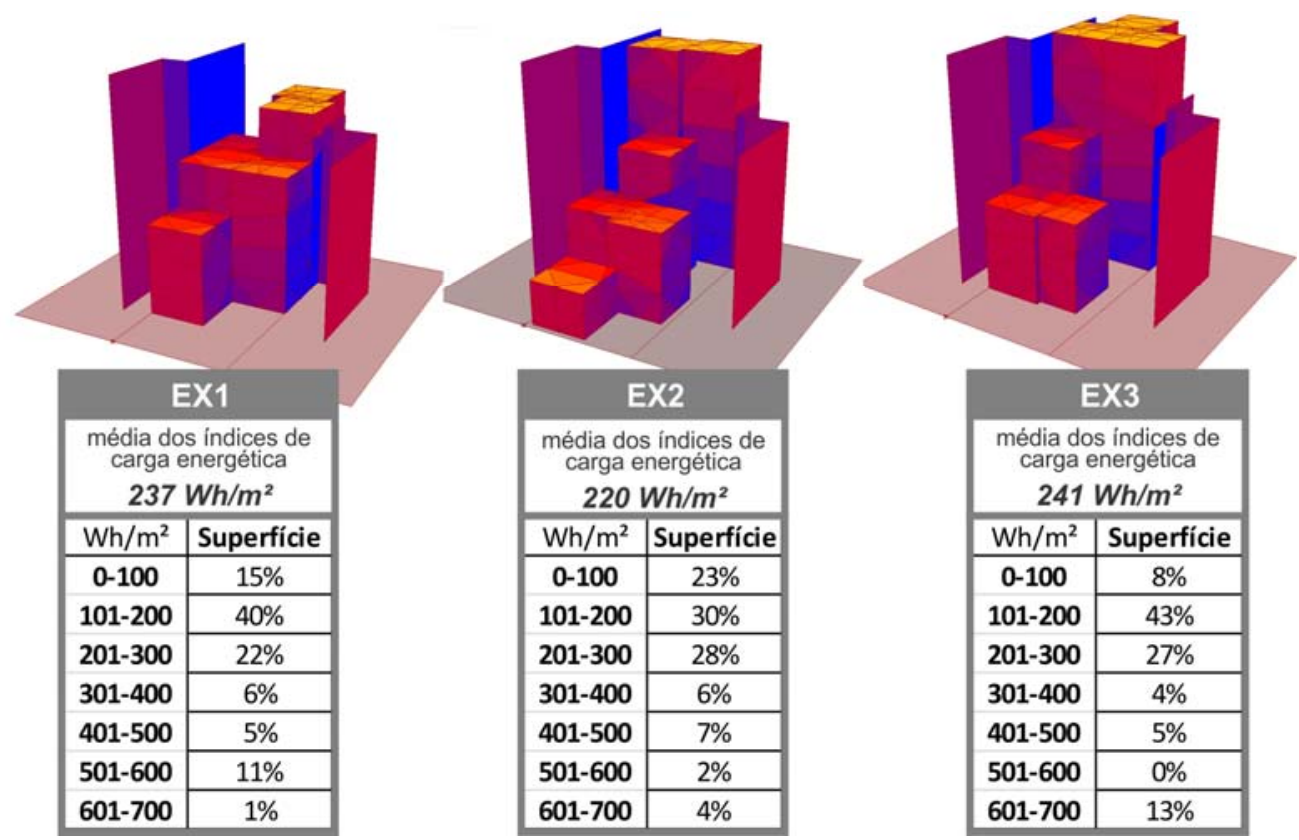

Fonte: elaborado pelo autor.

Isso não significa que todos os critérios serão atendidos em sua plenitude, pois diante de uma situação tradeoff, ou seja, onde há um conflito de escolha, ao favorecer o ganho de um dos objetivos pode ocorrer o sacrifício de outro, existindo assim uma "relação de compromisso" entre os diferentes objetivos. Isso pode ser verificado ao comparar a forma inicial e a final obitida no EX3 (Figura 12), não reduzindo a dimensão do perímetro, mas aumentando o volume total e a área de superfícies com maior carga energética (Figura 11), ocorrendo assim a negociação entre os objetivos.

Figura 12 - Análise entre a forma inicial e a final encontrada no experimento EX3

\section{Forma Inicial}

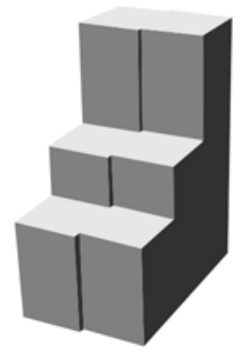

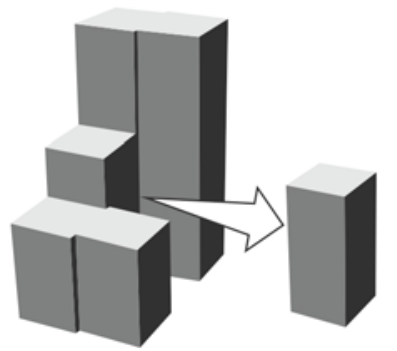
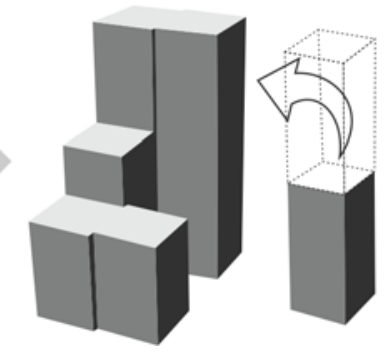

Forma Final

Fonte: elaborado pelo autor.

\section{CONCLUSÃO}

As atuais discussões sobre crise ambiental, gerando questões relacionadas à sustentabilidade tornam-se frequentes, e as certificações como LEED (Leadership in Energy and Environmental Design) e BREEAM (Building Research Establishment Environmental Assessment Methodology) são utilizadas para a regulamentação e o direcionamento do 
desenvolvimento de projetos sustentáveis. Dessa forma, a utilização do pensamento computacional integrado às ferramentas digitais para a definição de uma metodologia projetual facilita a incorporação de conhecimentos de outras áreas no processo de projeto, contribuindo para a obtenção de soluções mais eficientes. Isso possibilitou o desenvolvimento de metodologias que potencializam o processo de projeto, permitindo ao projetista criar diferentes abordagens capazes de gerarem maior aproximação com a realidade onde o projeto será implantando. Exemplo disso foi o algoritmo evolutivo apresentado neste artigo como uma técnica form-finding, utilizando os dados informacionais do sítio como critério para a modelagem volumétrica do edifício e cobertura. A possibilidade da simulação da realidade por meio da inserção e avaliação das soluções no seu contexto geográfico potencializa a metodologia de projeto, uma vez que as informações sobre o seu desempenho podem ser adotadas como um dos critérios responsáveis pela definição formal, obtendo mais eficiência desde a fase inicial de concepção projetual.

\section{AGRADECIMENTOS}

O autor agradece à FAPESP pela concessão da bolsa de doutorado, processo 2012/181127, e à Profa. Dra. Gabriela Celani pela orientação durante o desenvolvimento da tese, do qual este artigo faz parte.

\section{REFERÊNCIAS}

BENTLEY, P. J. Evolutionary Design by Computers. San Francisco: Morgan Kaufmann Publishers Inc, 1999.

FISCHER, T.; HERR, C. M. Teaching Generative Design. In: SODDU, C. (ed.). Proceedings of the 4th International Generative Art Conference. Generative Design Lab DIAP, Politecnico di Milano, dez. 2001. Disponível em: <http://www.generativeart.com>. Acesso em: 10 abr. 2014.

FLOREANO, D.; MATTIUSSI, C. Bio-inspired artificial intelligence: theories, methods, and technologies. Cambridge: The MIT Press, 2008.

KOLAREVIC, B. Towards Integrative Design. International Journal of Architectural Computing, v. 7, n. 3, p. 335-344, 2009.

; MALKAWI, A. (Ed.). Performative Architecture. New York: Routledge, 2005.

LORENZO-EIROA, P.; SPRECHER, A. (Ed.). Architecture in Formation: On the Nature of Information in Digital Architecture. Oxon: Routledge, 2013.

MITCHELL, M. An introduction to genetic algorithms. Cambridge: The MIT Press, 1999.

OXMAN, R. Theory and design in the first digital age. Design studies, v. 27, n. 3, p. 229-265, 2006.

TERZIDIS, K. Algorithmic architecture. Oxford: Elsevier, 2009.

YEANG, K.. Bioclimatic skyscrapers. 2ed. London: Ellipsis, 1994. 